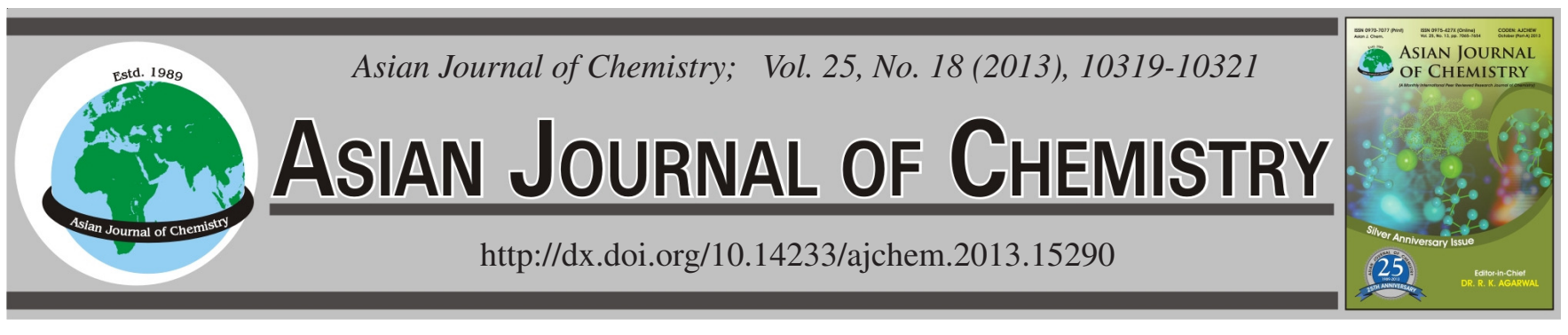

\title{
Sensitive Analysis of Azelnidipine and Related Derivative in Human Plasma by Ultra-Performance Liquid Chromatography-Tandam Mass Spectrometry
}

\author{
G. Suneetha ${ }^{1, *}$, P. Venkateswarlu ${ }^{1}$ and P.S.S. Prasad ${ }^{2}$
}

${ }^{1}$ Department of Chemistry, Sri Venkateswara University, Tirupati-517 502, India

${ }^{2}$ Department of Drugs Control Administration, Hyderabad-500 038, India

*Corresponding author: E-mail: sunithareddy04@gmail.com

(Received: 6 February 2013;

Accepted: 14 November 2013)

AJC-14392

\begin{abstract}
A rapid and sensitive method for analysis of azelnidipine in human plasma by ultra-performance liquid chromatography tandem mass spectrometry is presented. After pretreatment of a plasma sample by solid-phase extraction, azelnidipine was analyzed by the system with $\mathrm{a} \mathrm{C}_{18}$ column. This method gave satisfactory recovery rates, reproducibility and good linearity of calibration curve in the range of $0.01-10$ $\mathrm{ng} / \mathrm{mL}$ for quality control samples spiked with azelnidipine. The detection limit was as low as $1 \mathrm{pg} / \mathrm{mL}$. This method seems very useful in forensic and clinical toxicology and pharmacokinetic studies.
\end{abstract}

Key Words: Azelnidipine, Ethyl derivative of azelnidipine, UPLC, Human plasma.

\section{INTRODUCTION}

Azelnidipine (Fig. 1) ( \pm )-(3)-(1-diphenylmethylazetidin3-yl)-5-isopropyl-2-amino-1, 4-dihydro-6-methyl-4-(3nitrophenyl)-3,5-pyridine dicarboxylate, is a new dihydropyridine derivative with calcium antagonistic activity. Whereas all the existing dihydropyridine calcium blockers have two methyl groups located at the 2- and 6-positions of the dihydropyridine ring, one methyl group at the 2-position is substituted by an amino group in the azelnidipine molecule. After a conduct of a series of preclinical and clinical studies ${ }^{1-4}$, this drug was launched into the market as CALBLOCK® in Japan in 2003. Azelnidipine occurs as two enantiomers due to an asymmetric carbon at the 4-position of the 1,4-dihydropyridine ring. Numerous methods have been published for the quantitative determination of dihydropyridine calcium blockers in plasma, including gas chromatography combined with different detectors $^{5-7}$ or high performance liquid chromatography (HPLC) coupled with UV and MS/MS detection ${ }^{8-11}$. Regarding the simultaneous determination of the calcium antagonist and the metabolite, nicardipine and the aromatized nicardipine (pyridine metabolite) in human plasma have been measured by HPLC method ${ }^{12}$. A quantitative liquid chromatography of azelnidipine and related impurities, a novel dihydropyridine calcium channel blocker, in human plasma by ultra-performance liquid chromatography-tandam mass, method suitable for the routine analysis of azelnidipine. This paper describes a sensitive, specific and rapid method for analysis of azelnidipine

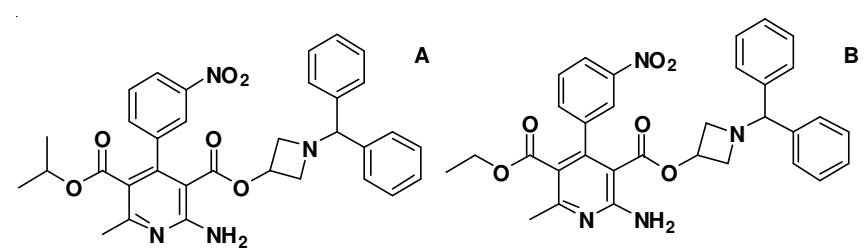

Fig. 1. Chemical structures of (A) azelnidipine and (B) Ethyl derivative of azelnidipine

and related impurities, a novel dihydropyridine calcium channel blocker, in human plasma by ultra-performance liquid chromatography-tandam mass.

Several articles described the pharmacokinetics of azelnidipine in Japanese volunteers ${ }^{13-16}$. But the pharmacokinetic profile of the drug in Chinese volunteers has not been reported. Recently, a new formulation of azelnidipine tablet was developed by Jiangsu Kanion Pharmaceutical Co., Ltd. (Lianyungang, China) and approved by State Food and Drug Administration of China to be put into phase I clinical trial. As entrusted by Jiangsu Kanion Pharmaceutical Co., Ltd., the investigation of the pharmacokinetics of the drug in Chinese volunteers was carried out. After an oral dose of $8 \mathrm{mg}$ the maximum plasma concentration of azelnidipine in adult humans is $5 \mathrm{ng} / \mathrm{mL}$.

\section{EXPERIMENTAL}

Azelnidipine and ethyl derivative of azelnidipine (IS) were donated by Chandan Laboratories Limited (Hyderabad, India). Acetonitrile and distilled water (DW) of the HPLC grade 
were purchased from Merck Research Laboratories India. Other common chemicals used were of the highest purity commercially available.

Solid-phase extraction procedure: The solid-phase extraction of azelnidipine and IS was performed essentially according to previous report ${ }^{12}$ with minor modifications. One $\mathrm{mL}$ volume of plasma containing or not containing azelnidipine was mixed with $5 \mathrm{ng}$ IS and $2 \mathrm{~mL}$ of $200 \mathrm{mM} \mathrm{HCl}$, vortexmixed for $1 \mathrm{~min}$ and centrifuged at $1600 \mathrm{rpm}$ for $5 \mathrm{~min}$. For solid-phase extraction, the supernatant fraction was applied to an Oasis HLB cartridge (60 mg, 3 cc; Waters Model Alliance 2489) preconditioned with $3 \mathrm{~mL}$ methanol and $3 \mathrm{~mL}$ distilled water. Each cartridge was washed with $3 \mathrm{~mL}$ distilled water. The columns were dried under vacuum for $10 \mathrm{~s}$. The analytes were eluted with $3 \mathrm{~mL}$ chloroform and the eluate was evaporated to dryness under nitrogen stream at room temperature. The residue was reconstituted in $25 \mu \mathrm{L}$ of $\mathrm{MeOH}$ and $75 \mu \mathrm{L}$ of $0.1 \%$ formic acid and vortex-mixed for $1 \mathrm{~min}$.

UPLC-MS/MS conditions: The liquid chromatography (LC) instrument used in combination with an MS/MS detector was a Waters Acquity UPLC system, including an Acquity UPLC binary pump and a sample manager (Waters). The column used for chromatographic separation was Acquity UPLC BEH $\mathrm{C}_{18}(50 \mathrm{~mm} \times 2.1 \mathrm{~mm}$. particle size $1.7 \mu \mathrm{m}$; waters $)$. The column temperature was maintained at $40^{\circ} \mathrm{C}$ and the following gradient system was used with a mobile phase A (20 mM ammonium acetate aqueous solution) and mobile phase B $(0.1 \%$ formic acid in acetonitrile) delivered at $0.5 \mathrm{~mL} / \mathrm{min}$. The linear gradient program was: $70 \% \mathrm{~A} / 30 \% \mathrm{~B}$ (0 min)-5\% A/95 \% B (4 min). The final mobile phase was used as a wash solvent to avoid any carry-over from previous injections. The auto sampler was maintained at $4{ }^{\circ} \mathrm{C}$ and the injection volume was $5 \mu \mathrm{L}$. The total run time for each sample analysis was $6 \mathrm{~min}$. The MS/ MS detection was made using peak areas in a positive ion mode on a tandem quadrupole mass spectrometer (Acquity TQD; Waters) equipped with electrospray ionization (ESI) interface. Quantitation was performed using selected reaction monitoring (SRM) with the transitions of $m / z, 580 \rightarrow 168$ for azelnidipine and $m / z 566.5 \rightarrow 168$ for IS. The optimal MS parameters were: capillary voltage, $3.0 \mathrm{kV}$; cone voltage, $30 \mathrm{~V}$; source temperature, $120^{\circ} \mathrm{C}$; desolvation temperature, $450{ }^{\circ} \mathrm{C}$; desolvation and cone gas, nitrogen with flow rates of 800 and $50 \mathrm{~L} / \mathrm{h}$, respectively; collision gas, argon with a flow rate of $0.15 \mathrm{~mL} / \mathrm{min}$; the optimized collision energies for azelnidipine and IS, $25 \mathrm{eV}$ each.

Human experiments: The assay was used to determine azelnidipine in plasma samples after administration a dose of $8 \mathrm{mg}$ azelnidipine to 12 healthy volunteers in a clinical study. The clinical study protocol was reviewed and approved. All volunteers were given written informed consent to participate in the study according to the principles of the declaration of Helsinki. Twelve healthy young volunteers participated in the study. For these 12 volunteers, their mean age was 20 years (range: 18-22 years); mean body weight was $59.5 \mathrm{~kg}$ (range: $52-71 \mathrm{~kg}$ ). Following an overnight fast, each volunteer received one tablet containing $8 \mathrm{mg}$ azelnidipine. Standard meals were provided after $4 \mathrm{~h}$ post-dose. Blood samples were collected pre-dose and at $0.5,1,1.5,2,3,4,6,9,12,24,48,72$ and $96 \mathrm{~h}$ post-dose. The azelnidipine plasma concentrations of these samples were determined and the pharmacokinetics of the drug in healthy young volunteers was evaluated. Model-independent pharmacokinetic parameters were calculated for azelnidipine. The maximum plasma concentration $\left(\mathrm{C}_{\max }\right)$ and the time to it $\left(\mathrm{t}_{\max }\right)$ were noted directly. The elimination rate constant $\left(\mathrm{k}_{\mathrm{el}}\right)$ was calculated by linear regression of the terminal points of the semi-log plot of plasma concentration against time. Elimination half-life $\left(t_{1 / 2}\right)$ was calculated using the formula $t_{1 / 2}=$ $0.693 / \mathrm{kel}$. The area under the plasma concentration-time curve AUC0-96 to the last measurable plasma concentration was calculated by the linear trapezoidal rule.

\section{RESULTS AND DISCUSSION}

Product ion mass spectra and selected reaction monitoring chromatograms: The UPLC-single stage mass spectrum obtained from azelnidipine and IS spiked into blank human plasma showed protonated molecular peaks at $\mathrm{m} / \mathrm{z}, 581.5$ and 567.5 , respectively, which appeared as base peaks. Other peaks appearing in their spectra were very small and thus could be neglected. The product ion mass spectra obtained from the protonated molecular ions of the authentic azelnidipine and IS are shown in Fig. 2. Base peaks at $m / z, 169$ appeared for both compounds. The peaks are probably formed by the cleavage in the middle of the bi-phenyl rings of each compound, which gave the ions of the same mass number at $\mathrm{m} / \mathrm{z}, 169$. Thus, we used this ion for quantitation of azelnidipine and IS. Fig. 3 shows SRM chromatograms for azelnidipine and IS spiked into blank human plasma at the concentration of $10 \mathrm{pg} / \mathrm{mL}$. Azelnidipine and IS appeared at 1.38 and $1.26 \mathrm{~min}$, respectively. There were no impurity peaks until 3 min of retention time.

Reliability of the method: The peak ratios of azelnidipine to IS obtained from SRM chromatograms were plotted for ten different concentrations against the concentration of azelnidipine in human plasma and a linear relationship was observed for azelnidipine in the range of $0.01-10 \mathrm{ng} / \mathrm{mL}$. The equation and its correlation coefficient were: $\mathrm{y}=0.296 \mathrm{xs}-0.001$ and $\mathrm{r}^{2}=$ 0.999. The limit of detection (LOD), defined as the concen-
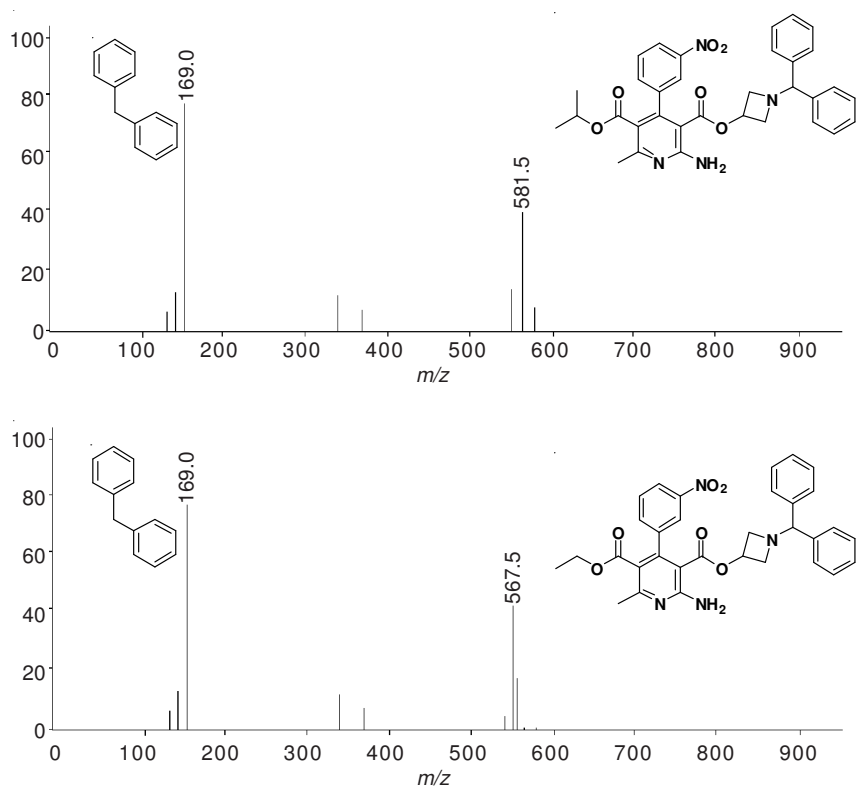

Fig. 2. Product ion mass spectra of azelnidipine and IS using precursor ions at $m / z 581.5$ and 567.5, respectively 

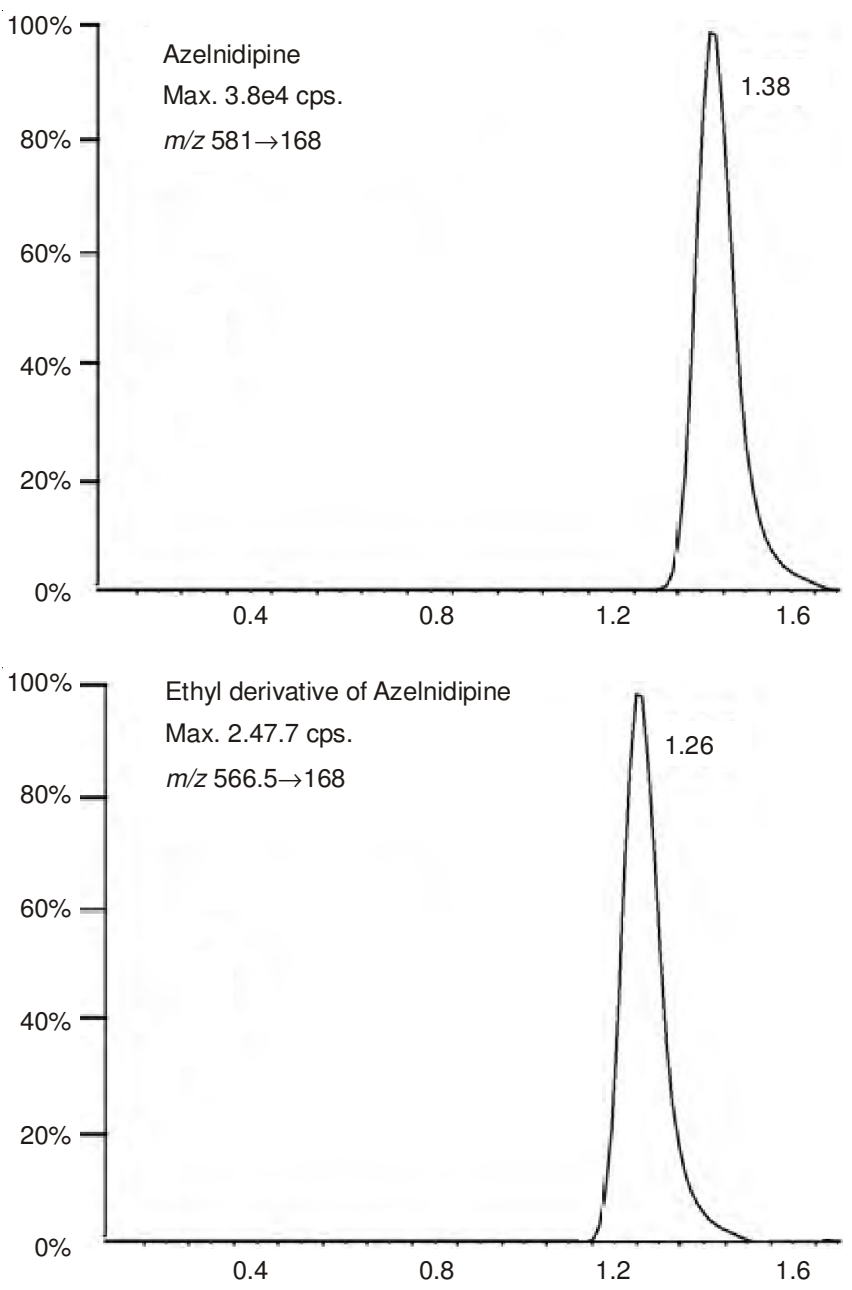

Fig. 3. Selected reaction monitoring (SRM) chromatograms for azelnidipine and IS spiked into blank human plasma obtained by ultra-performance liquid chromatography-tandem mass spectrometry. The amounts of azelnidipine and IS spiked into $1 \mathrm{~mL}$ plasma were $10 \mathrm{pg}$ and $5 \mathrm{ng}$, respectively

tration giving the signal-to-noise ratio of 3 , was $1 \mathrm{pg} / \mathrm{mL}$ with quality control samples of human plasma. The recovery rates of azelnidipine spiked into human plasma at concentrations of 0.01 and $10.0 \mathrm{ng} / \mathrm{mL}$ were $93.7 \pm 1.2$ and $93.7 \pm 1.1 \%(\mathrm{n}=$ 5 each), respectively. Table- 1 shows accuracy and precision data of the present method. Accuracy values were 86.9-103\%; precision values were not greater than $5.5 \%$.

TABLE-1

ACCURACY AND PRECISION DATA OF THE METHOD

\begin{tabular}{cccc}
\hline $\begin{array}{c}\text { Measurement } \\
\text { interval }\end{array}$ & $\begin{array}{c}\text { Concentration added } \\
(\mathrm{mg} / \mathrm{mL})\end{array}$ & $\begin{array}{c}\text { Accuracy } \\
(\%)\end{array}$ & $\begin{array}{c}\text { Precision }(\mathrm{CV} \\
\%)\end{array}$ \\
\hline \multirow{2}{*}{ Intra-day } & 0.01 & 87.7 & 4.9 \\
& 10.0 & 100.0 & 2.1 \\
\hline \multirow{2}{*}{ Inter day } & 0.01 & 86.9 & 5.5 \\
& 10.0 & 103.0 & 2.3 \\
\hline
\end{tabular}

In the first report describing analysis of azelnidipine in plasma by HPLC with fluorescence detection, the linearity was demonstrated in the range of $0.04-5 \mathrm{ng} / \mathrm{mL}$, but HPLC without MS cannot give the final identification of analytes. In the second report for analysis of the drug in human plasma by GC/MS, the linear range of the calibration curve was $0.5-20$ $\mathrm{ng} / \mathrm{mL}$ and the detection limit was $0.25 \mathrm{ng} / \mathrm{mL}$. The present UPLC-MS/MS method for azelnidipine is more than 50 times as sensitive as the GC/MS method.

Actual analysis of azelnidipine in human plasma after single oral dose: A 60-year-old volunteer ingested $10 \mathrm{mg}$ azelnidipine (therapeutic dose) and his blood was sampled 1.5 and $4 \mathrm{~h}$ after ingestion. For blood taken just before ingestion, no peak appeared at $1.38 \mathrm{~min}$ in an SRM chromatogram at $\mathrm{m} / \mathrm{z}$ 168.

\section{Conclusion}

To our best of knowledge, this is the first report describing LC-MS/MS analysis of azelnidipine. A sensitive LC-MS-MS method for the quantification of azelnidipine in human plasma was developed and validated. No significant interferences caused by endogenous compounds were observed. This method gave satisfactory recovery rates, reproducibility and good linearity of calibration curve in the range of $0.01-10.0 \mathrm{ng} / \mathrm{mL}$ for quality control samples spiked with azelnidipine. The detection limit was as low as $1 \mathrm{pg} / \mathrm{mL}$ and sensitive assay is suitable for the pharmacokinetic study and bioavailability evaluation of azelnidipine formulations and can also be used as a therapeutic drug monitoring method in clinic to check the plasma concentration of azelnidipine in hypertensive patients.

\section{ACKNOWLEDGEMENTS}

The authors are highly indebted to the authorities of S.V. University, Tirupati for providing laboratory facilities.

\section{REFERENCES}

1. K. Oizumi, H. Nishino, H. Koike, T. Sada, M. Miyamoto and T. Kimura, Jpn. J. Pharmacol., 51, 57 (1989).

2. K. Oizumi, H. Nishino, M. Miyamoto, J. Fukushige, M. Fukami and H. Koike, Jpn. J. Pharmacol., 51, 501 (1989).

3. N. Nakashima, K. Kawabata and K. Sasahara, J. Clin. Ther. Med., 16, 163 (2000).

4. N. Nakashima, K. Kawabata and K. Sasahara, J. Clin. Ther. Med., 16, 179 (2000).

5. N. Nakashima, K. Kawabata and K. Sasahara, J. Clin. Ther. Med., 16, 191 (2000).

6. Y. Tokuma, T. Fujiwara and H. Noguchi, J. Chromatogr. B, 345, 51 (1985).

7. B.J. Schmid, H.E. Perry and J.R. Idle, J. Chromatogr. B, 425, 107 (1988).

8. M. Nagasawa, H. Sasabe, T. Shimizu and H. Mori, J. Chromatogr. B, 577, 275 (1992).

9. M. Yriti, P. Parra, E. Iglesias and J.M. Barbanoj, J. Chromatogr. A, 870, 115 (2000).

10. I. Niopas and A.C. Daftsios, J. Pharm. Biomed. Anal., 32, 1213 (2003).

11. M. Pawula, D. Watson, T. Teramura, T. Watanabe, S. Higuchi and K.N. Cheng, J. Chromatogr. B Biomed. Sci. Appl., 719, 113 (1998).

12. L.H. Miglioranca, R.E. Barrientos-Astigarraga, B.S. Schug, H.H. Blume, A.S. Pereira and G. De Nucci, J. Chromatogr. B Anal. Technol. Biomed. Life Sci., 814, 217 (2005).

13. A.T. Wu, I.J. Massey and S. Kushinsky, J. Pharm. Sci., 73, 1444 (1984).

14. H. Koike, T. Kimura, T. Kawasaki, T. Sada, T. Ikeda, A. Sanbuissho and H. Saito, Ann. Rep. Sankyo Res. Lab., 54, 1 (2002).

15. K. Kuramoto, S. Ichikawa, A. Hirai, S. Kanada, T. Nakacachi and T. Ogihara, Hypertens. Res., 26, 201 (2003).

16. J. Ma, S. Kishida, G. Wang, K. Meguro, H. Imuta, H. Oonuma, H. Iida, T. Jo, H. Takano, T. Morita, R. Nagai and T. Nakajima, J. Cardiovasc. Pharmacol., 47, 314 (2006). 